\title{
Mean field study of structural changes in Pt isotopes with the Gogny interaction
}

\author{
R. Rodríguez-Guzmán, ${ }^{1,2}$ P. Sarriguren, ${ }^{2}$ L. M. Robledo, ${ }^{3}$ and J. E. García-Ramos ${ }^{1}$ \\ ${ }^{1}$ Departamento de Física Aplicada, Universidad de Huelva, E-21071 Huelva, Spain \\ ${ }^{2}$ Instituto de Estructura de la Materia, CSIC, Serrano 123, E-28006 Madrid, Spain \\ ${ }^{3}$ Departamento de Física Teórica C-XI, Universidad Autónoma de Madrid, E-28049 Madrid, Spain
}

(Received 2 December 2009; published 11 February 2010)

\begin{abstract}
The evolution of the nuclear shapes along the triaxial landscape is studied in the Pt isotopic chain using the self-consistent Hartree-Fock-Bogoliubov approximation based on the Gogny interaction. In addition to the parametrization D1S, the new incarnations D1N and D1M of this force are also included in our analysis to assess to which extent the predictions are independent of details of the effective interaction. The considered range of neutron numbers $88 \leqslant N \leqslant 126$ includes prolate, triaxial, oblate, and spherical ground-state shapes and serves as a detailed comparison of the predictions obtained with the new sets D1N and D1M against the ones provided by the standard parametrization Gogny-D1S in a region of the nuclear landscape for which experimental and theoretical fingerprints of shape transitions have been found. Structural evolution along the Pt chain is discussed in terms of the deformation dependence of single-particle energies.
\end{abstract}

DOI: 10.1103/PhysRevC.81.024310

PACS number(s): 21.60.Jz, 21.30.Fe, 27.70.+q, 27.80.+w

\section{INTRODUCTION}

Quadrupole collectivity is one of the most relevant features of nuclear structure $[1,2]$. In this context, the theoretical understanding of the evolution of the nuclear shapes, and the structural changes associated with it, represents an active research field [3-9]. From the experimental side, low-lying spectroscopy is one of the most powerful sources of information about nuclear shapes and shape transitions, since one can establish signatures correlating the excitation energies with the deformation properties [10-16].

Nowhere, however, is the evolution of nuclear shapes more documented or challenging than around the proton shell closure $Z=82$. For example, the neutron-deficient lead isotopes with neutron number $N \approx 104$ display three $0^{+}$states within $1 \mathrm{MeV}$ excitation energy [17]. The very rich and challenging variety of nuclear shapes also extends to the neighboring $\mathrm{Hg}$ and Po isotopes [10]. In particular, it has been demonstrated [18] that in the case of the lead isotopes, the decreasing trend observed in the binding energy difference $\delta_{2 p}(Z, N)=E(Z-2, N)-2 E(Z, N)+E(Z+2, N)$ for decreasing mass number $A$, can already be described quantitatively by mean field models in terms of deformed ground states of $\mathrm{Hg}$ and $\mathrm{Po}$ nuclei, while the inclusion of the quadrupole correlation energy [19] brings the calculations even closer to experiment. From the experimental point of view [10], the neutron-deficient mercury isotopes exhibit deformed ground states, while the situation is more involved in the case of Po nuclei (see, for example, Ref. [20] and references therein).

Considerable effort has also been devoted to characterizing Pt nuclei [12,13,21-29]. In this case, several deformation regimes have been suggested. Previous theoretical investigations [30-35] have found triaxial and oblate ground-state shapes for the heaviest Pt isotopes, while for the light ones a prolate deformed regime is predicted. From the experimental point of view $[14,16,36]$, the energy ratio $E_{4^{+}} / E_{2^{+}}$is almost 2.5 for $\mathrm{Pt}$ nuclei with neutron numbers $110 \leqslant N \leqslant 118$ already pointing to $\gamma$ soft shapes. The role played by the $\gamma$ degree of freedom in Pt isotopes has also been stressed by the comparison of experimental and theoretical results performed in Ref. [37], which shows that good agreement can be obtained if triaxiality is taken into account. Further down, a transition to a vibrational regime is suggested for ${ }^{168-172} \mathrm{Pt}$ by both the experimental data and their theoretical interpretation [22].

The shape evolution provided by the mean field framework [2], based on the most recent incarnations of the Gogny interaction [38], in the isotopic chain ${ }^{166-204} \mathrm{Pt}$ is considered in the present study as a representative sample of nuclei close to the $Z=82$ proton shell closure for which prolate, triaxial, oblate, and spherical ground-state shapes are found.

Nuclear shapes around the proton magic number $Z=82$ have been studied using a wide variety of theoretical models. Low-lying minima in both $\mathrm{Pb}$ and $\mathrm{Hg}$ isotopes have been predicted within the framework of the Strutinsky method [39-41]. From a mean field perspective, the coexistence between different nuclear shapes in $\mathrm{Pt}, \mathrm{Hg}$, and $\mathrm{Pb}$ nuclei has been considered within the relativistic mean field approximation $[42,43]$. Deformed ground states were predicted in the case of $\mathrm{Pb}$ isotopes as well as superdeformed ground states in $\mathrm{Hg}$ isotopes at variance with experiment. To cure this problem, a new parametrization of the relativistic mean field Lagrangian, called NLSC, was introduced in Ref. [44]. More recently, a new set called NL3* has been proposed in Ref. [45], providing an improved description of the ground-state properties of many nuclei such as $\mathrm{Pb}$ isotopes. Studies based on Skyrmelike models have been reported [46-50], while the shape coexistence in ${ }^{182-192} \mathrm{~Pb}$ was analyzed in Ref. [51] using the Hartree-Fock-Bogoliubov (HFB) approach based on the parametrization D1S [52] of the Gogny interaction [38]. From a beyond mean field perspective, symmetry projected configuration mixing, based on both Skyrme $[53,54]$ and Gogny [55] energy density functionals, has been successfully employed in this region of the nuclear chart, establishing a firm ground to support the experimental evidence for rotational bands in the neutron-deficient $\mathrm{Pb}$ isotopes built, on coexisting low-lying $0^{+}$states. On the other hand, evidence for $\gamma$ vibrations and shape evolution has also been considered in 
the nuclei ${ }^{184-190} \mathrm{Hg}$ [56], where a five-dimensional collective Hamiltonian was built with the help of constrained Gogny-D1S HFB calculations.

Just below the proton magic number $Z=82$, the nuclei with $A=170-200$ are particularly interesting, because small islands of oblate deformation might be favored energetically. Transitions from prolate to oblate shapes, as the number of neutrons increases, have been predicted in this mass region, using collective models [13], phenomenological Woods-Saxon or Nilsson potentials [33,57,58], and relativistic mean field [59-61] as well as non-relativistic deformed Hartree-Fock (HF) $[8,62]$ and HFB calculations [8,63]. Signatures for a transition from prolate to oblate ground states, as the number of neutrons increases from $N=110$ to $N=122$, have been found in $\mathrm{Hf}, \mathrm{W}$, and Os isotopes. In particular, such a transition was found [8] to happen at $N=116-118$. Subsequently, the evolution of the ground-state shapes along the triaxial landscape of several isotopes of $\mathrm{Yb}, \mathrm{Hf}, \mathrm{W}$, Os, and $\mathrm{Pt}$ has been studied in Ref. [63] within the framework of the mean field approximation based on both Skyrme and Gogny interactions. This region is also an active research field within the interacting boson model (IBM) [64-72].

Taking into account that around the neutron midshell $N=104$, examples of coexisting configurations have been found [10], it is very interesting to study the propagation of the nuclear shapes in the Pt isotopic chain and the way it can be correlated with the details in the underlying single-particle levels as functions of the deformation parameters. For such a task, the mean field approximation appears as a first tool, incorporating important correlations within the concept of spontaneous symmetry breaking and allowing a description of the evolution of shell structure and deformation all over the nuclear chart (see, for example, Refs. [5,7-9,63,73] and references therein).

In the present work, our study will be performed within the self-consistent HFB framework based on the Gogny interaction [38]. In addition to D1S [52], which is still the most standard and thoroughly tested parametrization, we also considered the two most recent parameter sets of the Gogny interaction, i.e., D1N [74] and D1M [75]. To the best of our knowledge, the results to be discussed later on in this paper, are the first systematic mean field study reported in the literature, using both $\mathrm{D} 1 \mathrm{~N}$ and D1M parametrizations to describe (mean field) ground-state properties of Pt nuclei. The selected isotopes ${ }^{166-204} \mathrm{Pt}$ cover almost the whole shell (i.e., $N=88-126$ ) and display a range of ground-state shapes wide enough to be considered a very challenging testing ground for comparing the (mean field) ground-state properties predicted with the new parametrizations D1N and D1M against the standard D1S Gogny functional. Our calculations also included the isotopes ${ }^{160-164} \mathrm{Pt}$, which turned out to be spherical and will not be further discussed in the present study.

The paper is organized as follows. In Sec. II we present a brief description of the theoretical framework used. The results of our study are discussed in Sec. III. There, we discuss, in Sec. III A, our Gogny-HFB calculations, for which axial symmetry is preserved as self-consistent symmetry, used to construct potential energy curves (PECs). In a second step, we discuss our study of the triaxial landscape, providing potential energy surfaces (PESs), by constraining on both $\beta$ and $\gamma$ quadrupole deformations. In our axial and triaxial HFB calculations, we considered at the same time the three parameter sets D1S, D1N, and D1M. The interaction Gogny$\mathrm{D} 1 \mathrm{~S}$, taken as a reference in the present study, is already considered as a global force able to describe reasonably well low-energy experimental data all over the nuclear chart (see, for example, Refs. [7,38,51,52,55,76-87] and references therein). This is also likely to be the situation with the new Gogny interactions D1N [63,74] and D1M [74], but still further explorations are required. Therefore, we consider all these interactions and functionals in the present study to assess to what extent the fine details of our mean field predictions for the nuclei ${ }^{166-204} \mathrm{Pt}$ are independent of the particular version of the Gogny force employed. After discussing the mean field systematics of deformation for the considered nuclei, we turn our attention, in Sec. III B, to the underlying single-particle properties as functions of both deformation (axial and triaxial) and mass number. This is relevant if one keeps in mind that from a mean field perspective, shape changes arise when the deformed single-particle levels are energetically favored to a different degree in open shell nuclei (the Jahn-Teller effect [88]). We also consider the behavior with neutron number of the spherical shell occupancies corresponding to the ground state of the different Pt isotopes in order to shed some light on the phenomena involved in the different deformation regimes. Finally, Sec. IV contains the concluding remarks and work perspectives.

\section{THEORETICAL FRAMEWORK}

To compute both PECs and PESs, we have used the (constrained) HFB method together with the parametrizations D1S, D1N, and D1M of the Gogny interaction. The solution of the HFB equations, leading to the vacuum $\left|\Phi_{\mathrm{HFB}}\right\rangle$, was based on the so-called gradient method $[63,89]$ to locate the minima. The kinetic energy of the center-of-mass motion has been subtracted from the Routhian to be minimized in order to ensure that the center of mass is kept at rest. The exchange Coulomb energy was considered in the Slater approximation, and we neglected the contribution of the Coulomb interaction to the pairing field.

The HFB quasiparticle operators have been expanded in a harmonic oscillator ( $\mathrm{HO}$ ) basis containing enough shells (i.e., $N=13$ major shells) to grant convergence for all values of the mass quadrupole operator and for all the nuclei studied. Energy contour plots will be shown in the $(Q, \gamma)$ plane [63] [instead of $(\beta, \gamma)]$ with

$$
\begin{aligned}
Q_{20} & =\frac{1}{2}\left\langle\Phi_{\mathrm{HFB}}\left|2 z^{2}-x^{2}-y^{2}\right| \Phi_{\mathrm{HFB}}\right\rangle, \\
Q_{22} & =\frac{\sqrt{3}}{2}\left\langle\Phi_{\mathrm{HFB}}\left|x^{2}-y^{2}\right| \Phi_{\mathrm{HFB}}\right\rangle, \\
Q & =\sqrt{Q_{20}^{2}+Q_{22}^{2}}, \\
\tan \gamma & =\frac{Q_{22}}{Q_{20}} .
\end{aligned}
$$


Other interesting pieces of information coming from the mean field are the single-particle energies (SPEs) for protons and neutrons. In our calculations, with the Gogny interaction, we are solving the HFB equations, and therefore the only quantities that can be properly defined are the quasiparticle energies. However, to have the more usual Nilssonlike diagrams, we have chosen to plot the eigenvalues of the Routhian [2] $h=t+\Gamma-\lambda_{20} Q_{20}-\lambda_{22} Q_{22}$, with $t$ being the kinetic energy operator, and $\Gamma$ the Hartree-Fock field. The term $\lambda_{20} Q_{20}+\lambda_{22} Q_{22}$ contains the Lagrange multipliers used to enforce the corresponding constraints. We have first performed calculations restricted to axially symmetric shapes, and in a second step triaxiality is included in our mean field analysis. In the first case, obviously, the term $\lambda_{22} Q_{22}$ is missing. In addition, the usual mean field constraints on both neutron and proton average numbers are taken into account. Parity and time reversal are self-consistent symmetries in our axial calculations, whereas parity and simplex are the ones imposed in the triaxial case [63].

\section{DISCUSSION OF THE RESULTS}

In this section, we discuss the results of the present study. The systematics of deformation obtained for the isotopes ${ }^{166-204} \mathrm{Pt}$ is described in Sec. III A. Single-particle properties are considered in Sec. III B.

\section{A. Mean field systematics of deformation for ${ }^{166-204} \mathbf{P t}$.}

The PECs obtained for the isotopes ${ }^{166-204} \mathrm{Pt}$ with our constrained Gogny-HFB calculations preserving axial symmetry are shown in Fig. 1 as functions of the quadrupole moment $Q_{20}$. Both prolate $\left(Q_{20}>0\right)$ and oblate $\left(Q_{20}<0\right)$ sides are displayed. The prolate side is equivalent to the triaxial results, to be discussed later on, with $Q=Q_{20}$ and $\gamma=0^{\circ}$, whereas the oblate side is equivalent to $Q=\left|Q_{20}\right|$ and $\gamma=60^{\circ}$.

As we can see, the interactions $\mathrm{D} 1 \mathrm{~N}$ and $\mathrm{D} 1 \mathrm{M}$ provide PECs which are extremely similar to the ones obtained with Gogny-D1S. The deformations of the oblate and prolate minima are practically independent of the force. The axial quadrupole moment $Q_{20}$ corresponding to the absolute minima of the PECs increases until $A \approx 180(N \approx 102)$, when it reaches the value $Q_{20} \approx 10$ b. A sudden prolate to oblate shape change occurs around $A=188$ for all the Gogny interactions considered in the present study. Beyond $A=190$, absolute oblate minima are obtained with quadrupole moments decreasing until the spherical shape is reached for ${ }^{204} \mathrm{Pt}$ ( $N=126)$. The opposite situation occurs with the secondary minima. On the other hand, the depth of both prolate and oblate wells (as compared to the spherical maximum) increases with increasing $N$ up to $A=182$ (roughly midshell), and it decreases from there on, the decrease being more pronounced for the prolate wells. This, as explained in Ref. [63], can be understood first as a consequence of the filling of downslopping levels coming from the high- $j$, unique parity $i_{13 / 2}$ neutron orbital, which would explain the increase of the depth, and then, at midshell, the filling of the up-slopping levels that would lead to the decrease of the height of the wells.
Slightly lower spherical barrier heights (i.e., the difference between the energy of the absolute minimum of the PEC and the energy of the spherical configuration) are predicted by the new Gogny forces D1N and D1M. Such a sensitivity of the spherical barriers with respect to details of the effective interactions used has already been found in previous studies $[8,9,49,90]$. In our calculations, the largest and smallest values of the total pairing energies corresponding to the spherical configurations are obtained with the sets D1M and $\mathrm{D} 1 \mathrm{~S}$, respectively. The set $\mathrm{D} 1 \mathrm{~N}$ provides pairing energies in between. This already reflects the different pairing content of the considered Gogny functionals, but we postpone a discussion on this point for later on.

The absolute values $\left|\Delta E_{p-o}\right|$ of the energy differences between the prolate and oblate minima of the PECs exhibit two bumps, the first with a maximum at $A=178$ (2.19, 1.97, and 2.11 MeV for D1S, D1N, and D1M) corresponds to isotopes with a prolate ground state, and the second one at $A=198(1.16,0.95$, and $0.99 \mathrm{MeV}$ for D1S, D1N, and D1M) corresponds to isotopes with an oblate ground state. The nucleus ${ }^{188} \mathrm{Pt}$, separating the two regions, has almost degenerate prolate and oblate minima with $\left|\Delta E_{p-o}\right|$ values of 45,111 , and $71 \mathrm{keV}$ for D1S, D1N, and D1M, respectively.

The previous axially symmetric results agree well with the ones in Ref. [8] using the parametrization SLy4 [91] of the Skyrme interaction in the particle-hole channel plus a zero range and density-dependent pairing interaction [92] (with strength $g=1000 \mathrm{MeV} \mathrm{\textrm {fm } ^ { 3 }}$ for both protons and neutrons) and with previous Skyrme-HF+BCS calculations with the parameter set SIII [30]. They also agree well with the results of the axial calculations reported in Ref. [61] using the parametrizations NL1 and NL2 of the relativistic mean field Lagrangian and with axial macroscopic-microscopic calculations reported in Ref. [34]. However, in our axial calculations, prolate and oblate minima lie quite close in energy $\left(\left|\Delta E_{p-o}\right| \leqslant 2.2 \mathrm{MeV}\right)$. Thus, a $\gamma$ path connecting them would be possible, and triaxiality could play a role, as will be discussed below, converting some of the axially symmetric minima into saddle points. Therefore, in a second step, we have also explored the triaxial landscape and construct PESs for all the considered nuclei.

The PESs obtained with the Gogny sets D1S, D1N, and D1M are shown in Figs. 2-4 in the form of $Q-\gamma$ planes. To simplify the presentation, the range of $Q$ values plotted is reduced to $0 \leqslant Q \leqslant 11 \mathrm{~b}$, and the contour lines are also severely reduced by considering contours every $250 \mathrm{keV}$ up to $2 \mathrm{MeV}$ higher than the energy of the minimum. We can see the spherical structure in ${ }^{204} \mathrm{Pt}$, while ${ }^{202-198} \mathrm{Pt}$ exhibit oblate $\left(\gamma=60^{\circ}\right)$ ground states with $Q \approx 3-4.5 \mathrm{~b}$. An island of triaxiality, centered around the nucleus ${ }^{188} \mathrm{Pt}$, is clearly visible from these figures. The ground-state triaxial coordinates $(Q, \gamma)$ within such an island of triaxiality evolve from $(Q \approx 5 \mathrm{~b}$, $\left.\gamma \approx 50^{\circ}-58^{\circ}\right)$ in ${ }^{196} \mathrm{Pt}$ to $\left(Q \approx 9 \mathrm{~b}, \gamma \approx 10^{\circ}\right)$ in ${ }^{184} \mathrm{Pt}$. The depth of these triaxial ground states, compared with the axial ones, is very small (see below). In the case of ${ }^{182-178} \mathrm{Pt}$, our calculations predict prolate $\left(\gamma=0^{\circ}\right)$ ground states with deformations $Q \approx 10$ b. Again, for ${ }^{176-172} \mathrm{Pt}$, very shallow triaxial minima are predicted. In the case of ${ }^{172} \mathrm{Pt}$, for example, 


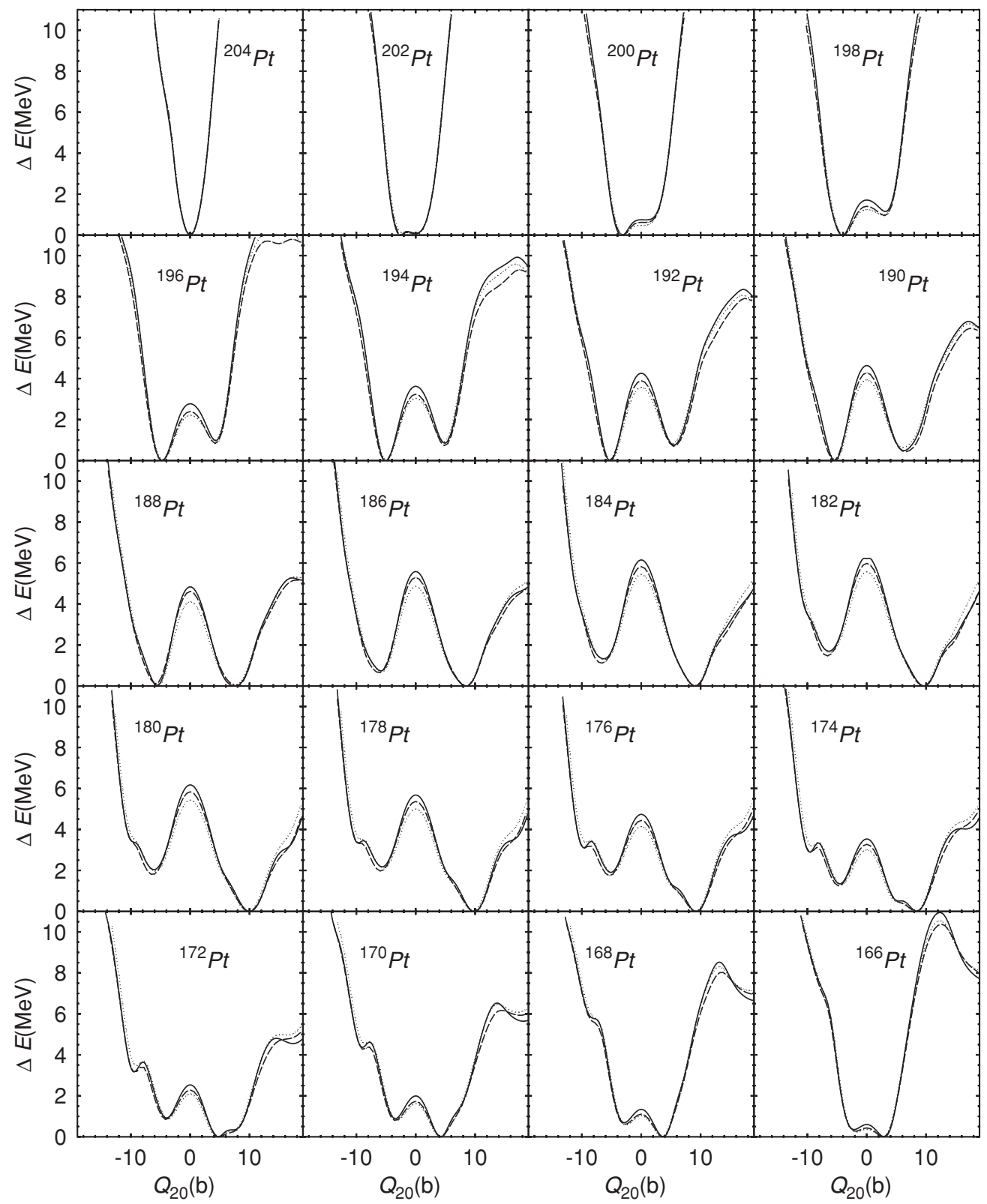

FIG. 1. Potential energy curves for the isotopes ${ }^{166-204} \mathrm{Pt}$ as functions of the axial quadrupole moment $Q_{20}$ calculated with the parametrizations D1S (continuous line), D1N (dashed line), and D1M (dotted line) of the Gogny interaction.

we find $\left(Q \approx 5 \mathrm{~b}, \gamma \approx 20^{\circ}\right.$ ). The isotopes ${ }^{170-166} \mathrm{Pt}$ display prolate ground states with $Q \approx 3-4.5 \mathrm{~b}$.

To obtain a more quantitative understanding of the PESs, we have plotted in Fig. 5 the mean field energies corresponding to the lowest axial minima $Q=Q_{20}$ of the PECs in Fig. 1 as functions of the deformation parameter $\gamma$. We observe that only one of the two axial minima remains in most of the cases. The nuclei ${ }^{202-198} \mathrm{Pt}$ exhibit oblate $\left(\gamma=60^{\circ}\right)$ absolute minima, and the prolate (axial) solutions become saddle points with excitation energies $\Delta E \leqslant 2 \mathrm{MeV}$. On the other hand, ${ }^{196-188} \mathrm{Pt}$ are rather $\gamma$ soft, with triaxial minima almost degenerate with the (axially symmetric) prolate and oblate solutions. In the case of ${ }^{188} \mathrm{Pt}$, for example, we obtain $|\Delta E|_{\text {triaxial-saddle }} \leqslant 0.9 \mathrm{MeV}$. Still inside the island of triaxiality, the oblate configurations in both ${ }^{186,184} \mathrm{Pt}$ already show the tendency to increase their excitation energies. A similar trend for the oblate solutions is observed within the mass range $182 \leqslant A \leqslant 174$. Oblate and prolate configurations for the nuclei ${ }^{172-166} \mathrm{Pt}$ are quite close $(0.3 \leqslant \Delta E \leqslant 1.2 \mathrm{MeV})$ and softly linked along the $\gamma$ direction.

A detailed account of the evolution of the ground-state triaxial coordinates $(Q, \gamma)$ as functions of the mass number $A$ is presented in the left panel of Fig. 6 for the sake of completeness. The striking similarity of the ground-state deformations obtained with the Gogny interactions D1S, D1N, and D1M becomes evident from this plot. We observe the emergence of weakly oblate ground states for the isotopes ${ }^{202-198} \mathrm{Pt}$. On the other hand, the sudden shape transition 

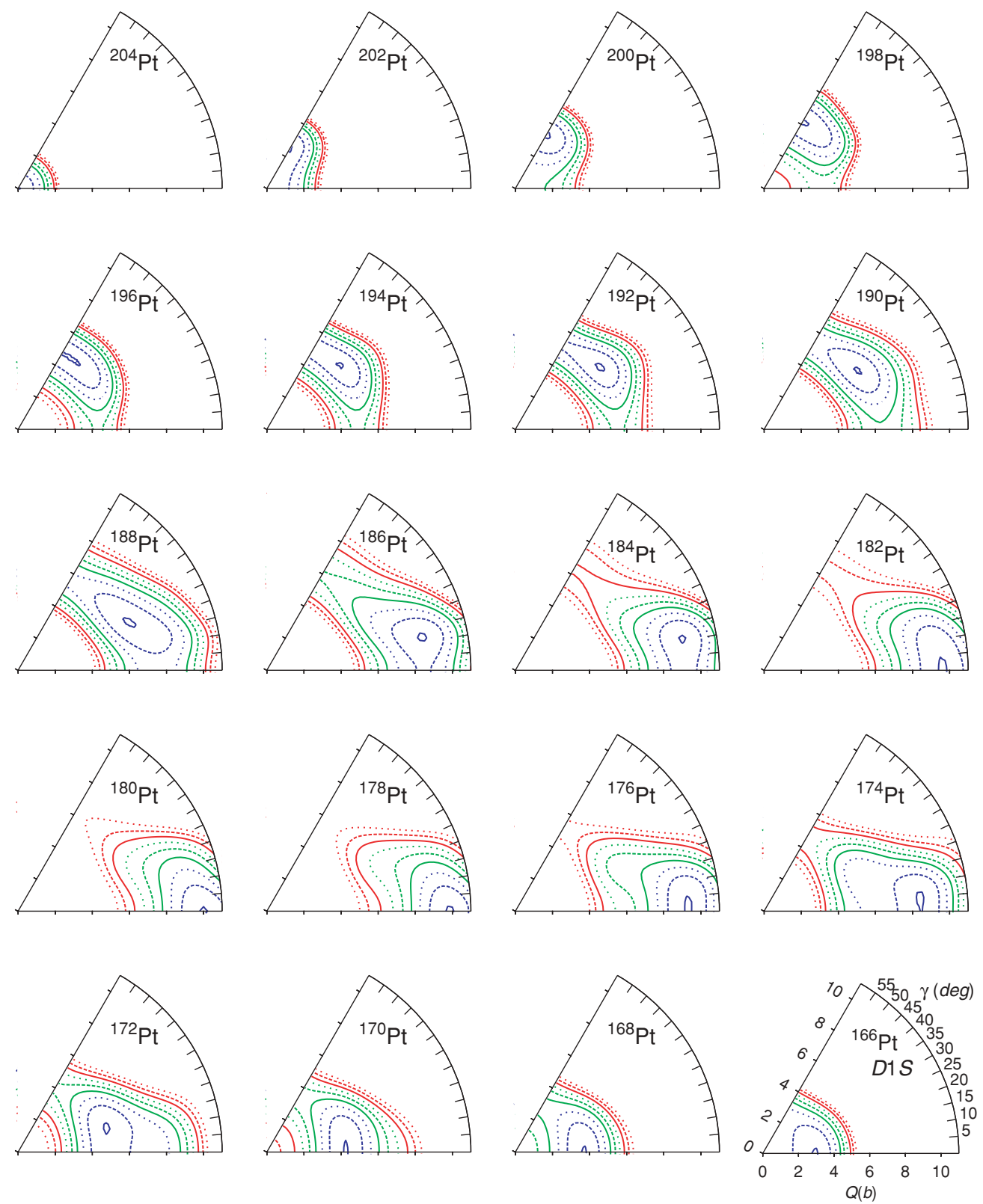

FIG. 2. (Color online) $Q-\gamma$ planes computed with the Gogny-D1S force for the isotopes ${ }^{166-204} \mathrm{Pt}$. The range of $Q$ values has been reduced to focus on the interval around the minima. The contour lines extend from the minimum up to $2 \mathrm{MeV}$ in steps of $0.25 \mathrm{MeV}$. Blue (black) contours are the three lowest, green (light gray) ones the next three, and red (dark gray) contours correspond to the three with higher energies. For each color, the solid line corresponds to the lower energy, dashed to the next contour, and dotted to the higher energy. The minimum of the triaxial landscape can be identified by the small ellipse surrounding it.

observed in the framework of the axially symmetric HFB calculations is now replaced by a smooth shape change through the island of triaxiality represented, in our case, by the isotopes ${ }^{184-196} \mathrm{Pt}$. A prolate deformed regime is predicted for the isotopes ${ }^{178-182} \mathrm{Pt}$. We find that the trend of shape changes predicted by our calculations in the considered $\mathrm{Pt}$ isotopes agrees well with the ones obtained in Refs. [31,32] and the conclusions extracted from the combination of total Routhian surface calculations plus quasiparticle random-phase approximation (TRS+QRPA) and IBM models in Ref. [22].
The general trend in our calculations is also consistent with results obtained in the framework of the Strutinsky approach $[33,35]$. Further down in neutron number, the TRS results $[3,93]$ predict a rapid change to a triaxial shape also found in our calculations around ${ }^{172} \mathrm{Pt}$. Finally, our PESs for ${ }^{170-166} \mathrm{Pt}$ exhibit features that could be interpreted as the onset of a more pronounced vibrational character for these nuclei [22].

The rather involved behavior of the neutron and proton pairing energies $E_{p p}$ (with opposite sign) versus mass number for the Pt isotopes is shown in the middle panel of Fig. 6. As 

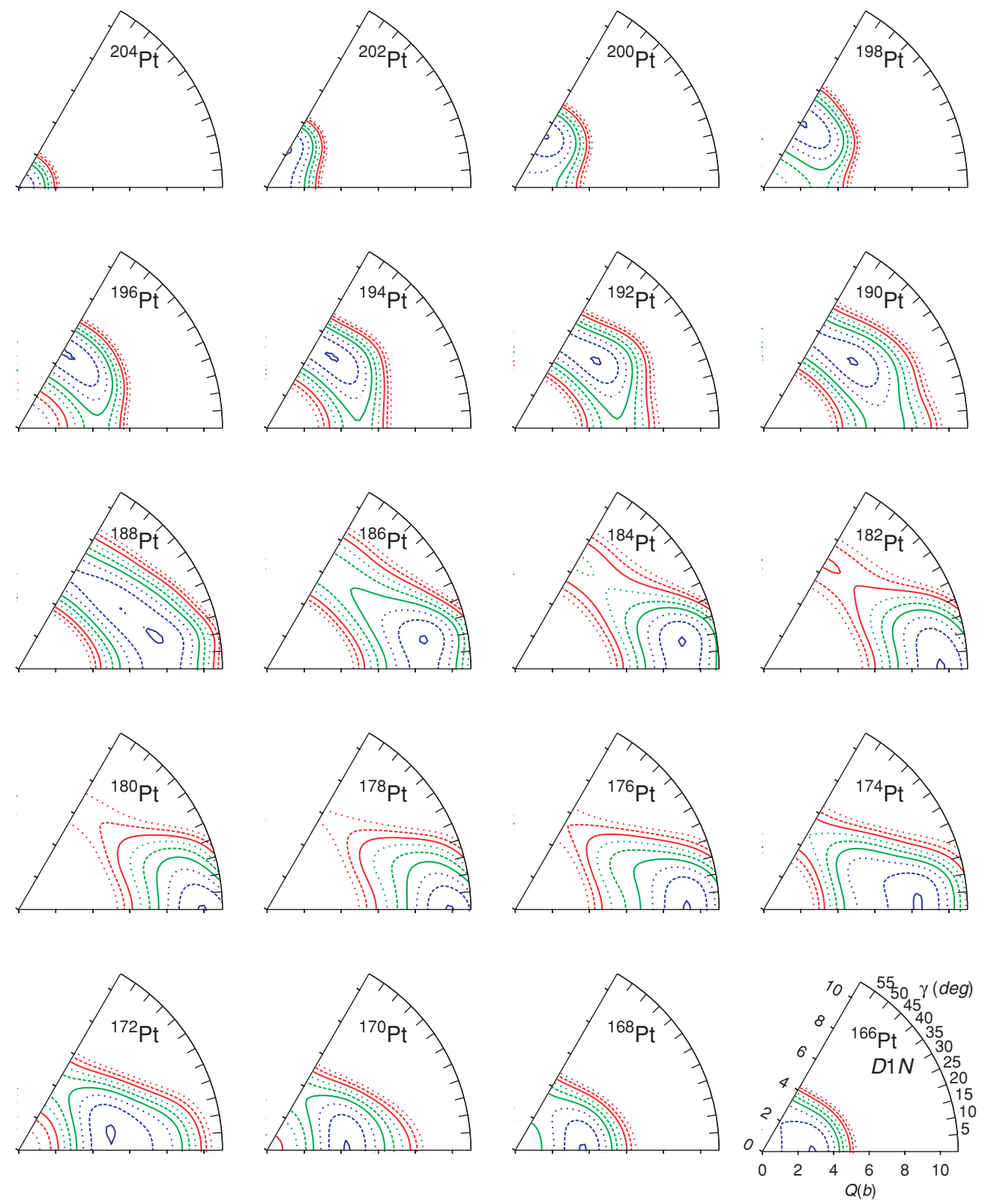

FIG. 3. (Color online) Same as Fig. 2, but for the Gogny-D1N force.

expected for a pure $N=126$ shell closure, neutron pairing collapses for the nucleus ${ }^{204} \mathrm{Pt}$. We observe that proton pairing shows a nonconstant behavior in spite of having a constant proton number $(Z=78)$ that comes from self-consistency effects. On the other hand, neutron pairing energies are lower inside the region between $A=174$ and $A=186$, which is precisely where the strong prolate deformation develops. The lowering of pairing energies is a consequence of the lowering of the level density that is needed (Jahn-Teller effect) to induce the deformed minima. For other values of $A$, the neutron level density around the Fermi level is higher, and, as a consequence, pairing correlations are stronger. Concerning different values of the neutron and proton pairing, for the three Gogny functionals the pairing energies follow the same isotopic trend, and the only relevant change is in the absolute value, which tends to be slightly lower for D1S. At this point it is worth remembering that the value of the pairing energy shown is related to the amount of pairing correlations present in the system, but it is by far not certain that the correlation is linear, in other words, the different values of $E_{p p}$ for different interactions do not necessarily imply the same quantitative behavior for pairing correlations.

Finally, the Thouless-Valatin moments of inertia $J^{(1)}=$ $3 /\left(E_{2^{+}}-E_{0^{+}}\right)$of the first $2^{+}$states in ${ }^{166-204} \mathrm{Pt}$ are plotted in the right panel of Fig. 6 as functions of the mass number $A$. They are compared with the experimental values extracted from the available systematics for the excitation energies of the first $2^{+}$states in ${ }^{166-204} \mathrm{Pt}$ (see, for example, Ref. [22]). The 

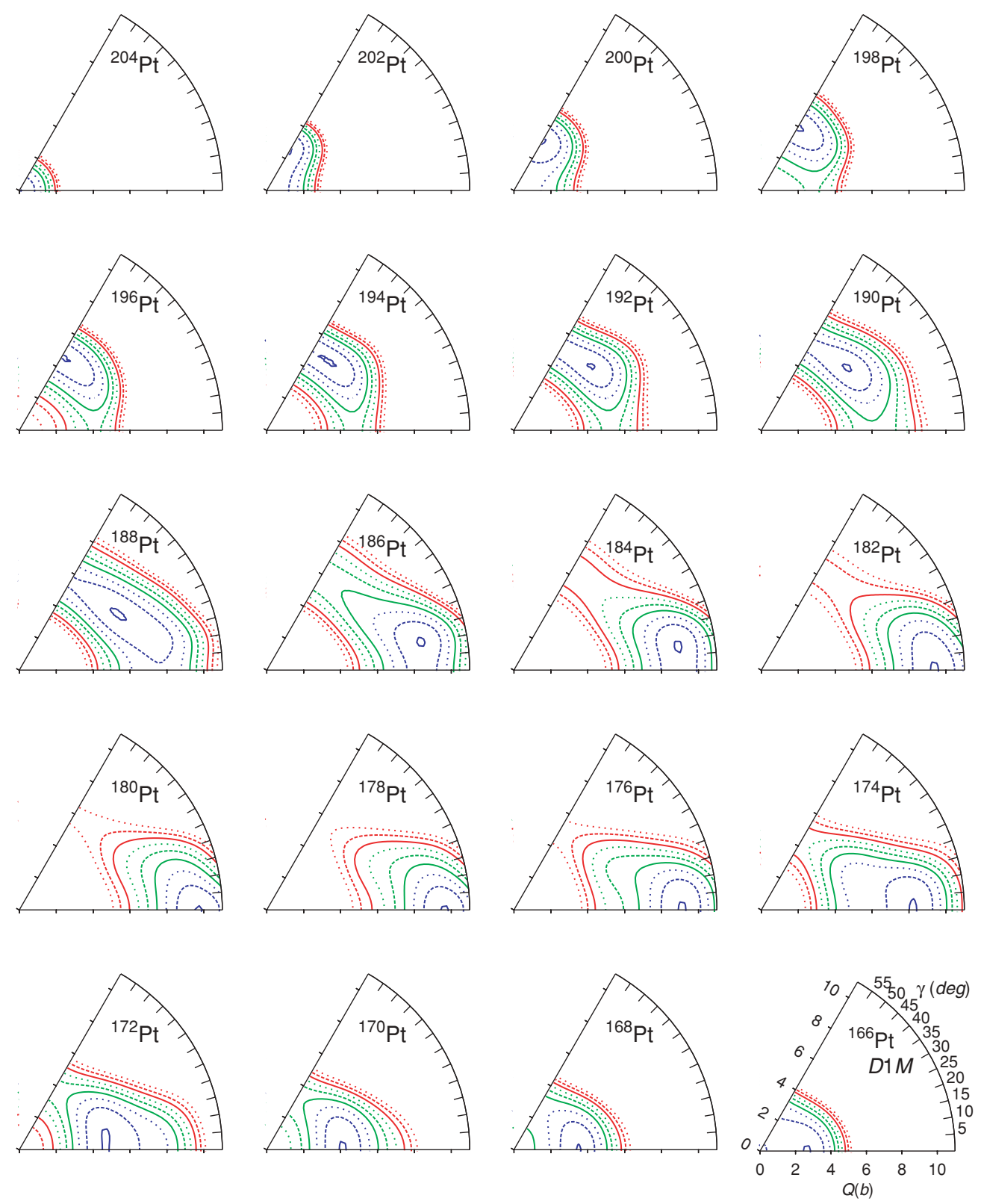

FIG. 4. (Color online) Same as Fig. 2, but for the Gogny-D1M force.

energies needed for the computation of $J^{(1)}$ have been obtained using the self-consistent cranking approximation introducing the usual time-reversal breaking term $-\omega J_{x}$ and the subsidiary condition $\left\langle J_{x}\right\rangle=\sqrt{I(I+1)}[2,63,81]$. The Thouless-Valatin moments of inertia strongly depend on pairing, and therefore a comparison of the results obtained with the three Gogny functionals considered in this work, which exhibit different pairing contents, can also give a hint of the quality of their predictions. As can be seen, the results follow the same isotopic trend irrespective of the Gogny force, with the tendency to be the largest for Gogny-D1S and the smallest in the case of Gogny-D1M. Nevertheless, the differences in the predicted values can still be attached to the uncertainties in the effective interactions, and we observe how the self-consistent cranking results tend to overestimate the experimental values. This defect, well known already, is a direct consequence of too low pairing at the mean field level, and its solution would require an improved treatment of pairing correlations. There are many mechanisms beyond mean field that modify the amount of pairing correlations in a given nuclear system, but there are two particularly important ones that tend to increase correlations. One is the restoration of the particle-number symmetry broken by the HFB method, and the other is the shape fluctuations around the HFB minimum. The latter is connected with the fact that the HFB minimum corresponds to a low level density region of the single-particle spectrum (see next subsection), and therefore its amount of pairing correlations is far lower than that of the neighboring configurations. Taking into 

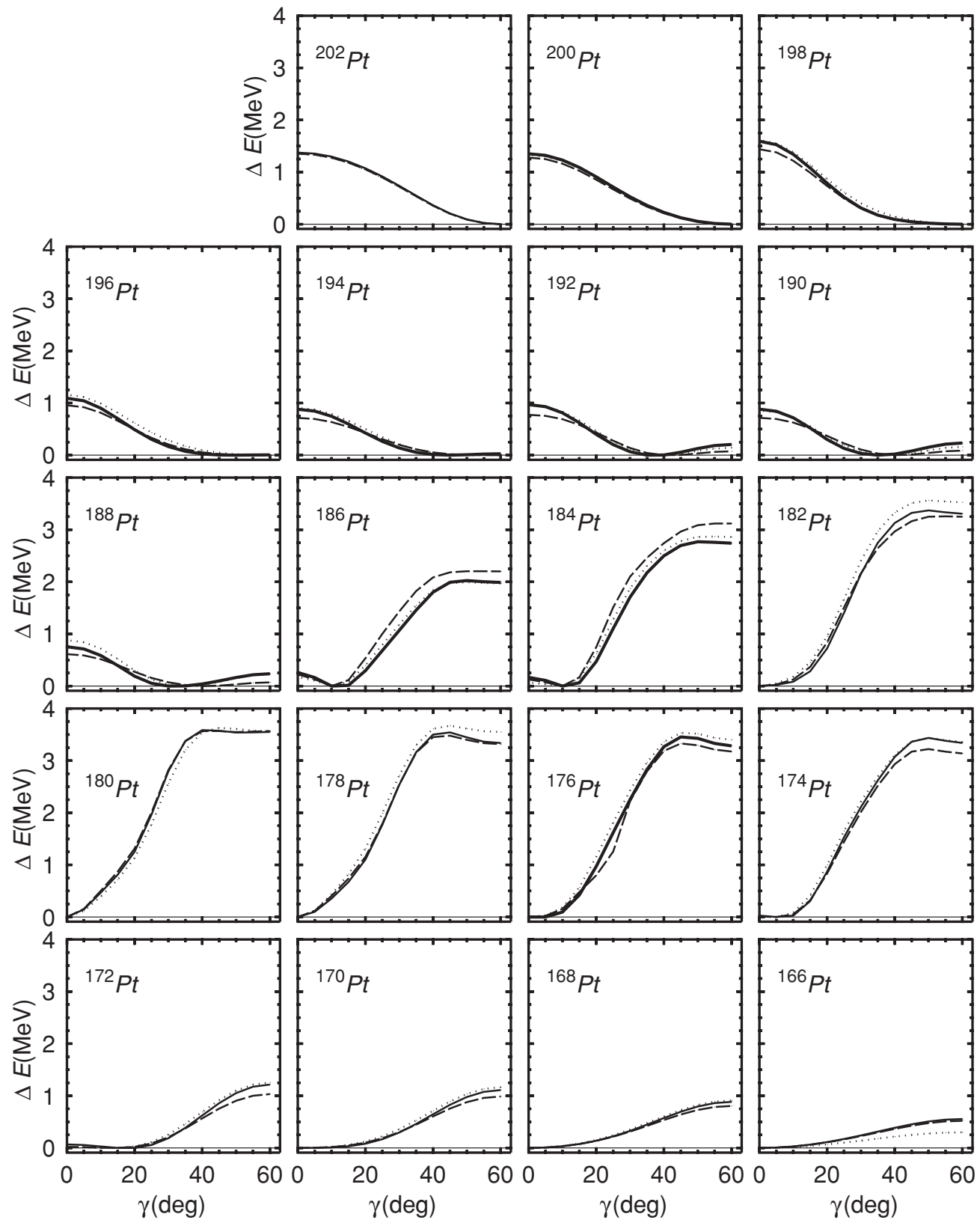

FIG. 5. Mean field excitation energies $\Delta E$ computed with the Gogny interaction D1S (continuous line), D1N (dashed line), and D1M (dotted line) are displayed as functions of the deformation parameter $\gamma$ for fixed values of the quadrupole moment $Q$ corresponding to the lowest minima of the axially symmetric calculations (see Fig. 1). Results for ${ }^{204} \mathrm{Pt}$ are not included because of the presence of a spherical ground state.

account the first mechanism would involve particle-number projection, whereas the second can only be treated in the scope of the GCM with the quadrupole moment as the generating coordinate or in the Bohr Hamiltonian method [87]. Clearly, both methods are out of the scope of the present study. On the other hand, we observe a certain correlation between the evolution with the number of neutrons of the quadrupole moments $Q$ and the moments of inertia $J^{(1)}$, as it is apparent by looking at the left and right panels of Fig. 6. This correlation is not so evident for the $\gamma$ degree of freedom.
The results discussed in this section indicate that the new interactions and functionals $\mathrm{D} 1 \mathrm{~N}$ and $\mathrm{D} 1 \mathrm{M}$ provide the same quality of mean field ground-state predictions for the considered Pt isotopes as the Gogny-D1S force taken as a reference in our calculations. This coincidence give us confidence in the robustness of our mean field predictions with respect to a change in the particular parametrization of the Gogny interaction used. The agreement is also rather good with the mean field picture obtained in Refs. $[8,63]$ within the HF+BCS framework based on the Skyrme parametrization SLy4 in the particle-hole channel plus a zero range and 

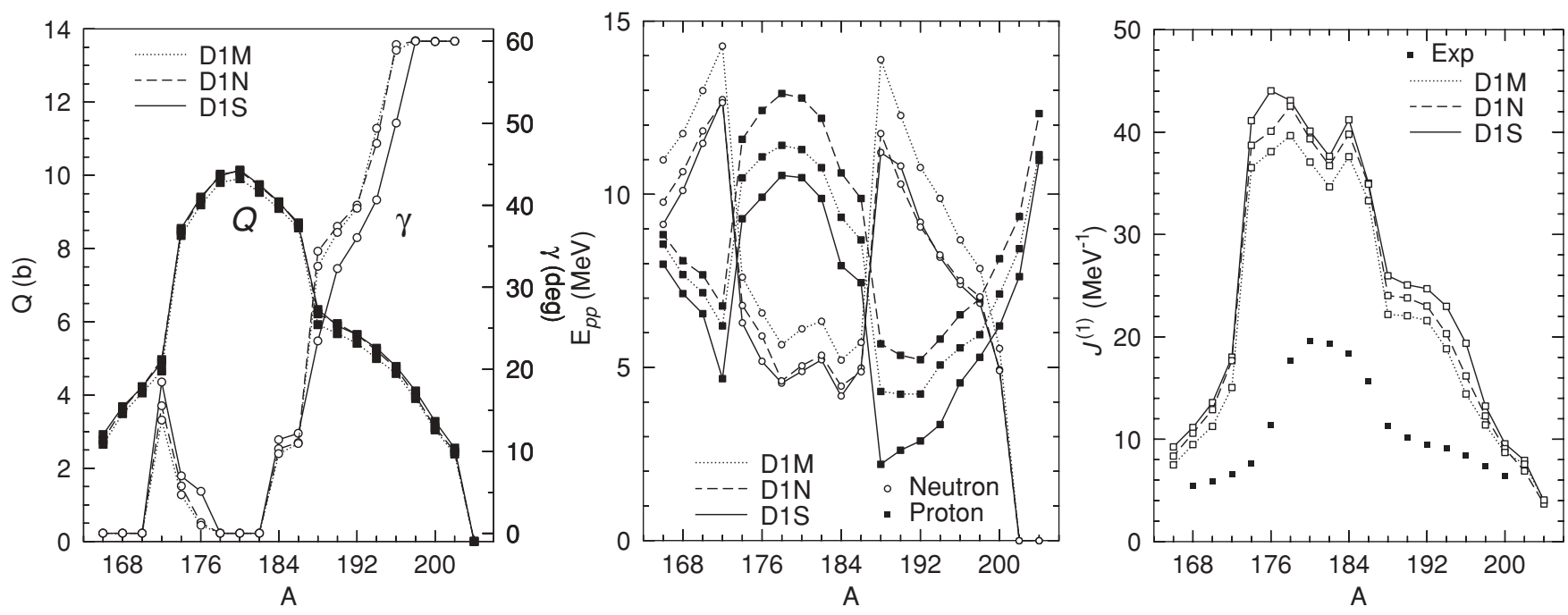

FIG. 6. Ground-state triaxial coordinates $(Q, \gamma)$ (left panel), neutron and proton pairing energies (middle panel), and Thouless-Valatin moments of inertia (right panel) are plotted as functions of the mass number A. Calculations have been performed with the Gogny interactions D1S, D1N, and D1M.

density-dependent pairing (with strength $g=1000 \mathrm{MeV} \mathrm{fm}^{3}$ for both protons and neutrons). Both Gogny-D1S and SkyrmeSLy4 represent well-reputed interactions whose reasonable predictive power has already been thoroughly tested all over the nuclear chart, and it is very satisfying to observe how the new parametrizations D1N and D1M, in spite of the relaxation of some of the original constraints in their fitting protocols and their being more oriented to reproducing nuclear masses [52,74], still follow very closely the fine details predicted with Gogny-D1S, Skyrme-SLy4, and other theoretical models $[8,22,30-33,61,63,93]$ for an isotopic chain with such a challenging shape evolution.

Let us remark that we are perfectly aware of the fact that the (static) mean field picture described above should also be extended to a dynamical treatment of the relevant degrees of freedom. This becomes clear from the topology of the PESs indicating that in order to access a quantitative comparison of the energy spectra and reduced transition probabilities with the considered Gogny interactions, the dynamical interplay between the zero point motion associated with the restoration of broken symmetries (mainly, angular momentum and particle number) and fluctuations in the collective parameters $(\beta, \gamma)$ should be taken into account. For such a cumbersome and computer-power-demanding extension, the Gaussian overlap approximation (GOA) appears as a first suitable choice $[82,94,95]$. For a very recent and excellent pedagogical review, the reader is also referred to Ref. [96]. Work along these lines is in progress and will be reported elsewhere.

\section{B. Single-particle properties}

In this section, we pay attention to single-particle properties of the considered isotopes. To this end, we first show in Fig. 7 SPE plots as functions of the axial quadrupole moment $Q_{20}$. The isotopes ${ }^{180} \mathrm{Pt},{ }^{188} \mathrm{Pt}$, and ${ }^{202} \mathrm{Pt}$ are taken as illustrative examples. The SPEs correspond to our Gogny-D1S calculations. For other nuclei and Gogny interactions, the results are quite similar.

The energy levels in Fig. 7 gather together around the spherical configuration $Q_{20}=0$, forming the spherical shell model orbitals $n l j$. Due to axial and time-reversal symmetries, SPE levels, tagged by the $K$ quantum number corresponding to the third component of the angular momentum in the intrinsic frame, are doubly degenerate. Positive and negative parity states are plotted with full and dashed lines, respectively. The proton $\lambda_{Z}$ and neutron $\lambda_{N}$ Fermi levels are also shown with a thick (red) dotted line. As it is well known, atomic nuclei "avoid" regions with high single-particle level densities (Jahn-Teller effect), and therefore the plots of SPEs versus deformation help us to identify regions where energy gaps favor the appearance of deformed minima [97].

We also look at the onset of deformation in the considered Pt nuclei using the Federman-Pittel (FP) criteria [98]. The origin of nuclear deformation is certainly a much more complicated phenomenon involving different mechanisms (see, for example, Refs. $[4,51,99,100]$ and references therein for detailed discussions on this issue). Even though the FP argument may be incomplete, it is certainly playing a role in the onset of nuclear deformation, and we resort to it in the present mean field study as a way to establish a very qualitative and simplified overall picture of shape changes in terms of the evolution of the underlying SPEs. For recent studies using similar ideas, see Refs. [63,101]. To incorporate the terminology of spherical orbitals to discuss deformed configurations, we assign to a given deformed single-particle orbital the label of the spherical orbit from which it originates at $Q_{20}=0$ [63].

In Fig. 7, we can see at zero deformation the spherical proton shells $3 s_{1 / 2}, 1 h_{11 / 2}, 2 d_{3 / 2}$, and $2 d_{5 / 2}$ below the Fermi level $\lambda_{Z}$ and the $1 h_{9 / 2}$ level above it. The relative position of $\lambda_{Z}$, with respect to the SPEs, is quite stable. The main effect of the different neutron numbers appears in the scale of Fermi energies $\lambda_{Z}$ ranging from around $-9 \mathrm{MeV}$ in the case of ${ }^{204} \mathrm{Pt}$ 

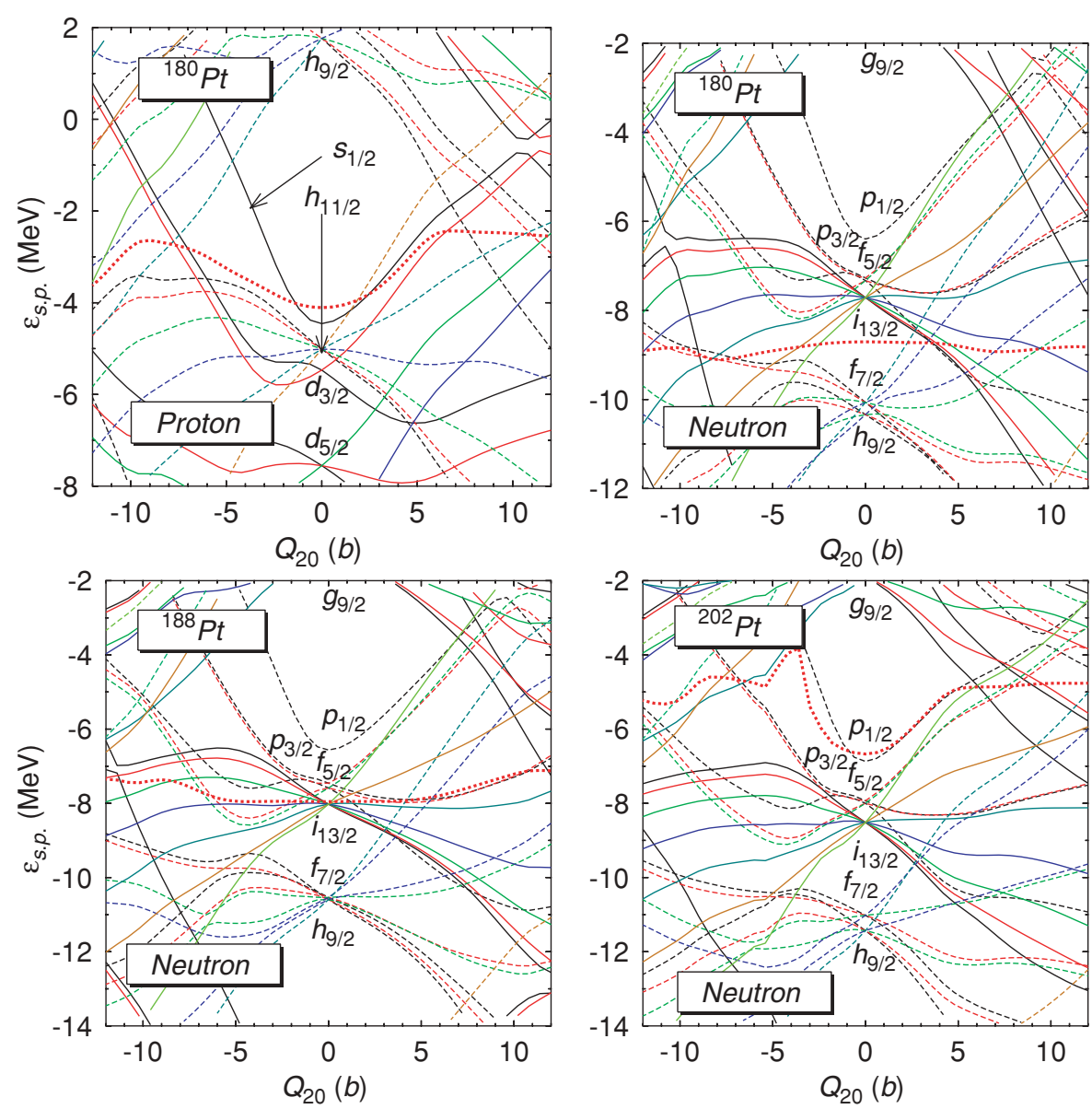

FIG. 7. (Color online) Upper panels: Proton and neutron SPEs for the nucleus ${ }^{180} \mathrm{Pt}$ as functions of the axial quadrupole moment $Q_{20}$. The Fermi levels are also plotted with a thick (red) dotted line. The results correspond to the force Gogny D1S. Solid (dashed) lines are used for positive (negative) parity states. With increasing $K=1 / 2,3 / 2,5 / 2, \ldots$ values, color labels are black, red, green, blue, dark-blue, brown, dark-green, etc. Lower panel: Same as above, but for neutron SPEs of the nuclei ${ }^{188} \mathrm{Pt}$ and ${ }^{202} \mathrm{Pt}$. The spherical quantum numbers at $Q_{20}=0$ are given for a number of orbitals close to the Fermi level. to values close to zero in the case of the neutron-deficient isotope ${ }^{166} \mathrm{Pt}$. Therefore, in Fig. 7 we have only plotted the proton SPEs for the isotope ${ }^{180} \mathrm{Pt}$. The closeness to the proton magic number $Z=82$ makes $\mathrm{Pt}$ isotopes $(Z=78)$ display a tendency to be spherical or slightly oblate, as can be seen in Fig. 7 from the huge spherical gap of $\sim 6 \mathrm{MeV}$ and the large gap of $\sim 3 \mathrm{MeV}$ on the oblate side centered around $Q_{20}=-5 \mathrm{~b}$.

For neutrons, the relevant spherical orbitals shown in Fig. 7 are $1 h_{9 / 2}, 2 f_{7 / 2}, 1 i_{13 / 2}, 2 f_{5 / 2}, 3 p_{3 / 2}, 3 p_{1 / 2}$, and $1 g_{9 / 2}$. Notice also that in the case of ${ }^{204} \mathrm{Pt}$, the tendency to be spherical is reinforced by its magic neutron number $N=126$, and the final result is a spherical nucleus. In our calculations, the $N=126$ spherical shell gap is observed to change with mass number from $\sim 5.5 \mathrm{MeV}$ in ${ }^{180} \mathrm{Pt}$ to $\sim 4.5 \mathrm{MeV}$ in ${ }^{202} \mathrm{Pt}$ going through $\sim 5 \mathrm{MeV}$ for ${ }^{188} \mathrm{Pt}$.

Between ${ }^{202} \mathrm{Pt}$ and ${ }^{196} \mathrm{Pt}, \lambda_{N}$ crosses a region on the oblate side, where an energy gap of $\sim 3 \mathrm{MeV}$ (i.e., half the size of the spherical gap) is found. This occurs at around $Q_{20}=$ $-5 \mathrm{~b}$, which overlaps perfectly with the gap on the oblate proton sector already mentioned above. On the other hand, as can be seen from Fig. 7 , the $1 i_{13 / 2}$ gets more and more occupied for increasing neutron number $N$, and at a certain point (i.e., beyond ${ }^{194} \mathrm{Pt}$ ) its role is transferred to the $2 f_{5 / 2}$ and $3 p_{3 / 2}$ orbitals. According to FP, only the neutron $2 f_{5 / 2}$ can interact with the proton $2 d_{3 / 2}$ (i.e., $n_{p}=n_{n}, l_{p}=l_{n}-1$ ); but since the $l$ values are in this case low, we should not expect a very strong interaction among them. This qualitatively explains the appearance of the very soft and close-to-spherical, oblate minima displayed in Fig. 1 within the mass range $196 \leqslant$ $A \leqslant 202$. A similar mechanism leads to very shallow and weakly prolate secondary minima in ${ }^{196,198} \mathrm{Pt}$.

Between ${ }^{194} \mathrm{Pt}$ and ${ }^{184} \mathrm{Pt}$, the neutron Fermi level on the prolate sector crosses a region with energy gaps of $\sim 3 \mathrm{MeV}$ and between $Q_{20}=3$ and $10 \mathrm{~b}$. From the neutron SPE plot of ${ }^{188} \mathrm{Pt}$, we observe how within this mass range, the most prominent role is played by the $1 i_{13 / 2}$ which, according to the FP criteria, interacts optimally with the proton $1 h_{11 / 2}$ to favor a prolate shape. On the oblate side, the interaction between the relevant orbitals, leading to the corresponding minima, takes place around $Q_{20}=-5 \mathrm{~b}$. The final net effect of these driving forces is the appearance of prolate and oblate minima that become saddle points inside the island of triaxiality (see also the discussion below).

Below ${ }^{182} \mathrm{Pt}$, our axially symmetric calculations predict strong prolate deformations. In this case, there is a strong gap on the neutron sector centered at $Q_{20}=10 \mathrm{~b}$, as can be seen from the neutron SPE plot of ${ }^{180} \mathrm{Pt}$. The most active deformed orbitals are the ones coming from the neutron $1 i_{13 / 2}$ and $2 f_{7 / 2}$. They interact with the deformed proton orbitals coming from the $1 h_{11 / 2}$ and $2 d_{5 / 2}$ to produce strongly prolate deformed shapes. The secondary oblate minima predicted for these nuclei can be associated with the proton and neutron energy gaps observed around $Q_{20}=-5$ b. Further down in 


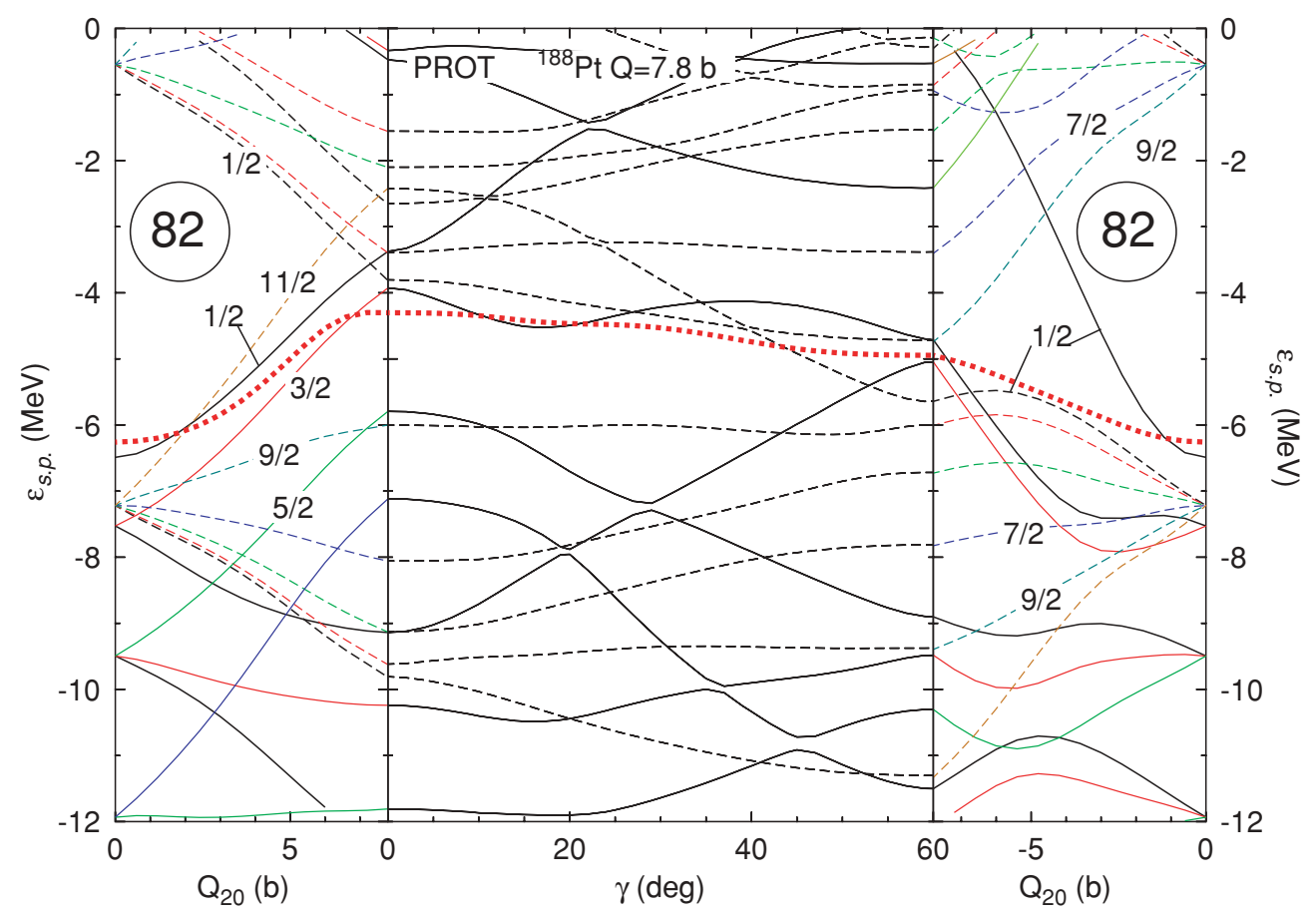

FIG. 8. (Color online) Proton SPEs for the nucleus ${ }^{188} \mathrm{Pt}$. In the left panel, the SPEs are plotted as a function of the axially symmetric quadrupole moment $Q_{20}$ from $Q_{20}=0$ up to $Q_{20}=7.8 \mathrm{~b}$. In the middle, the triaxial proton SPEs are plotted as functions of the deformation parameter $\gamma$ and for $Q=7.8 \mathrm{~b}$. Finally, on the right, the axially symmetric SPEs are plotted as a function of $Q_{20}$ from $Q_{20}=-7.8 \mathrm{~b}$ up to $Q_{20}=0$. The proton Fermi level is depicted as a thick (red) dotted line. Some relevant $K$ values are also included. In the axial plots, at $Q_{20}=0$ (i.e., sphericity) we have the $d_{5 / 2}$ at $\epsilon \approx-9.5 \mathrm{MeV}$; at an energy of around $-7.5 \mathrm{MeV}$, we have the $d_{3 / 2}$; and at $\epsilon \approx-7.2 \mathrm{MeV}$, we have the $h_{11 / 2}$ orbital. Also at $Q_{20}=0$, the $Z=82$ shell gap is clearly visible. Solid (dashed) lines are used for positive (negative) parity states. For more details, see the main text.

neutron number, the neutron Fermi level $\lambda_{N}$ explores regions lower in energy, resulting in less pronounced prolate minima located around $Q_{20}=4 \mathrm{~b}$ in the neutron-deficient isotopes ${ }^{166,168} \mathrm{Pt}$.

Let us now turn our attention to the origin of triaxiality. For this, proton and neutron SPE plots are shown in Figs. 8 and 9, respectively, as functions of the triaxial deformation parameter $\gamma[6,63]$ for the nucleus ${ }^{188} \mathrm{Pt}$. We consider $Q=7.8 \mathrm{~b}$, which corresponds to a rather large region in the $Q-\gamma$ plane near the triaxial minimum (located at $Q=6.2 \mathrm{~b}$, see left panel of Fig. 6), where the PES is very flat in the two coordinates (see Fig. 2). The value of $Q=7.8 \mathrm{~b}$ also corresponds to the position of the axial prolate minimum (see Fig. 1). These plots allow us to identify the $K$ values of the triaxial SPEs at the prolate $\left(\gamma=0^{\circ}\right)$ and oblate $\left(\gamma=60^{\circ}\right)$ limits and the change of the $K$ contents observed in most of the levels as $\gamma$ evolves. Typical examples in this context are the negative parity $K=1 / 2$ proton level with $\mathrm{SPE} \epsilon \approx-3 \mathrm{MeV}$ at $Q=7.8 \mathrm{~b}$ and $\gamma=0^{\circ}$, which transforms into a $K=9 / 2$ level at $\gamma=60^{\circ}$ and the positive parity $K=13 / 2$ neutron level with SPE $\epsilon \approx-4.2$ $\mathrm{MeV}$ at $Q=7.8 \mathrm{~b}$ and $\gamma=0^{\circ}$ which transforms into a $K=$ $1 / 2$ level at $\gamma=60^{\circ}$. The rather low level density below the proton Fermi level for $\gamma$ between $0^{\circ}$ and $30^{\circ}$ favors the flatness of the energy curve as a function of $\gamma$, helping thereby the development of the triaxial minimum in ${ }^{188} \mathrm{Pt}$ around $\gamma=30^{\circ}$. From this plot we also conclude that the isotopes with two protons less (Os isotopes) will be more prone to triaxiality, as discussed in Ref. [63]. Concerning the behavior of the neutron SPEs with $\gamma$, depicted in Fig. 9, we observe a region of low level density in the interval of $\gamma$ between $0^{\circ}$ and $20^{\circ}$ that would favor the development of triaxiality. From there on, the level density increases; therefore, to develop a triaxial minimum, the system is forced to change its $Q$ deformation to lower values (see Fig. 2 for the nucleus under consideration, ${ }^{188} \mathrm{Pt}$ ). The removal of two or four neutrons makes the level density in the range of $\gamma$ between $0^{\circ}$ and $20^{\circ}$ even lower than in the case of ${ }^{188} \mathrm{Pt}$, explaining the $\gamma$ deformed minima observed in Fig. 5 for the nuclei ${ }^{184,186} \mathrm{Pt}$. On the other hand, the addition of two or four extra neutrons leads to a decrease of the level density in the interval between $\gamma \approx 30^{\circ}$ and $\gamma=60^{\circ}$, favoring the appearance of triaxial minima in ${ }^{190,192} \mathrm{Pt}$ and flat curves in ${ }^{194,196} \mathrm{Pt}$, as observed in Fig. 5.

In the spirit of the shell model, it is also interesting to compute the spherical occupancies $v(l j, Q, \gamma)$ of the different $l j$ orbitals in the (usually) deformed ground states $\left|\Phi_{\mathrm{HFB}}(Q, \gamma)\right\rangle$ of the Pt isotopes studied in this paper. They are given by

$$
v(l j, Q, \gamma)=\sum_{n} \sum_{m}\left\langle\Phi_{\mathrm{HFB}}(Q, \gamma)\left|c_{n l j m}^{+} c_{n l j m}\right| \Phi_{\mathrm{HFB}}(Q, \gamma)\right\rangle,
$$

where $c_{n l j m}^{+}$and $c_{n l j m}$ are the creation and annihilation operators of spherical harmonic oscillator orbits characterized by the quantum numbers $n, l, j, m$. The sum in $m$ is introduced 


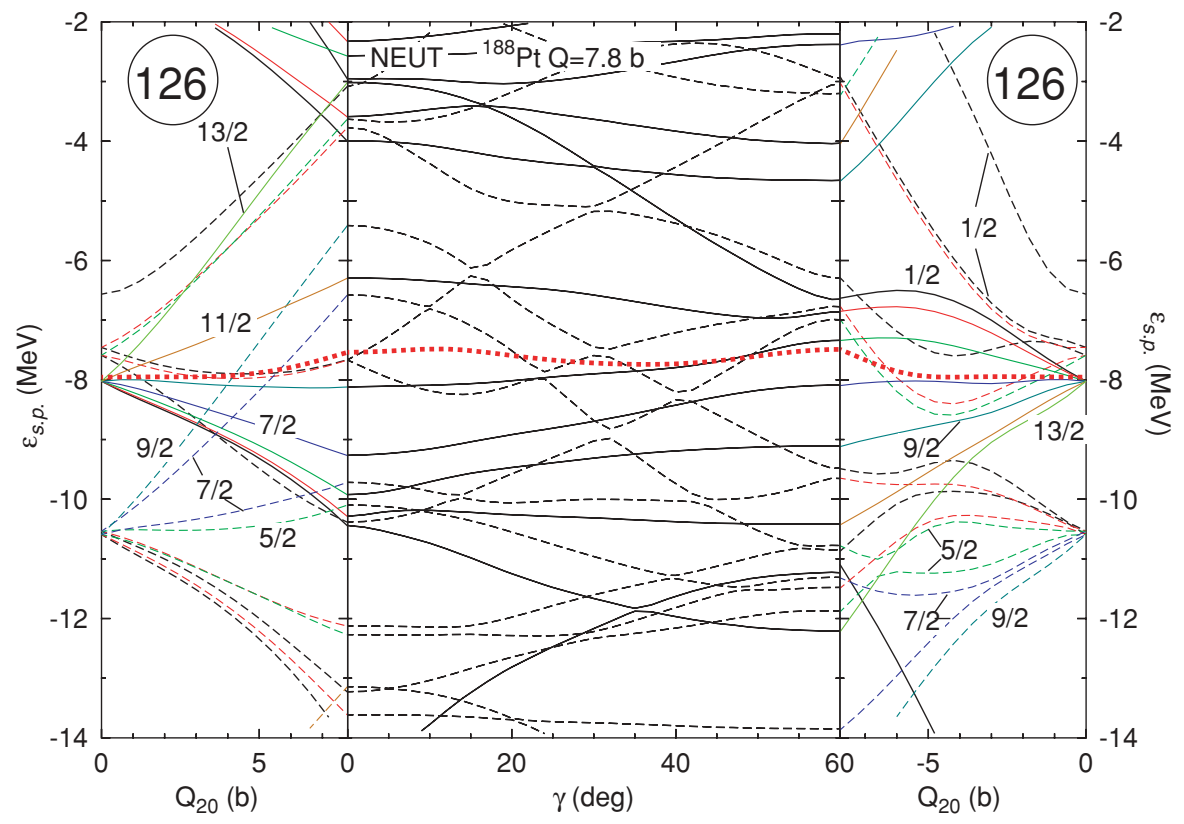

FIG. 9. (Color online) Same as Fig. 8, but for neutrons. In the axial plots, at $Q_{20}=0$ (i.e., sphericity) we have the $h_{9 / 2}$ and $f_{7 / 2}$ at $\epsilon \approx-10.5 \mathrm{MeV}$; at an energy of around $-8 \mathrm{MeV}$, we have the $i_{13 / 2}$; and at $\epsilon \approx-7.5 \mathrm{MeV}$, we have the $f_{5 / 2}$ and $p_{3 / 2}$ orbitals. Also at $Q_{20}=0$, the $N=126$ shell gap is clearly visible. to make the quantity (5) invariant under changes in orientation (and therefore to represent a genuine "spherical" quantity). The sum in the radial quantum number $n$ does not allow us to pin down which specific $\mathrm{HO}$ orbital is occupied; but, on the other hand, it allows us to get rid of the uncertainties associated with the fact that the radial wave function of the nuclear orbitals is close but not exactly the one of the HO.

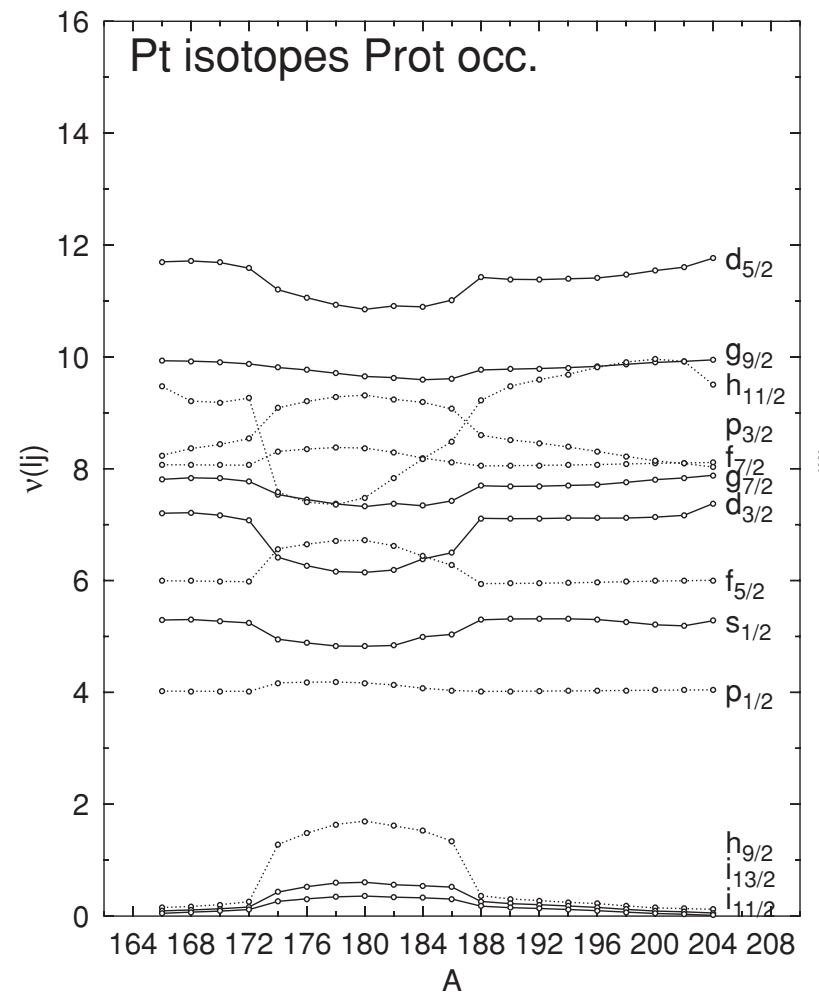

The proton spherical occupancies in the ground-state wave functions of all the Pt isotopes considered are shown on the left panel of Fig. 10. As the number of protons remains constant along the isotopic chain, one could expect a flat behavior of all the occupancies. However, we observe in that figure how the occupancies of the different orbitals can be classified in two different regimes, namely, the weak deformation regime

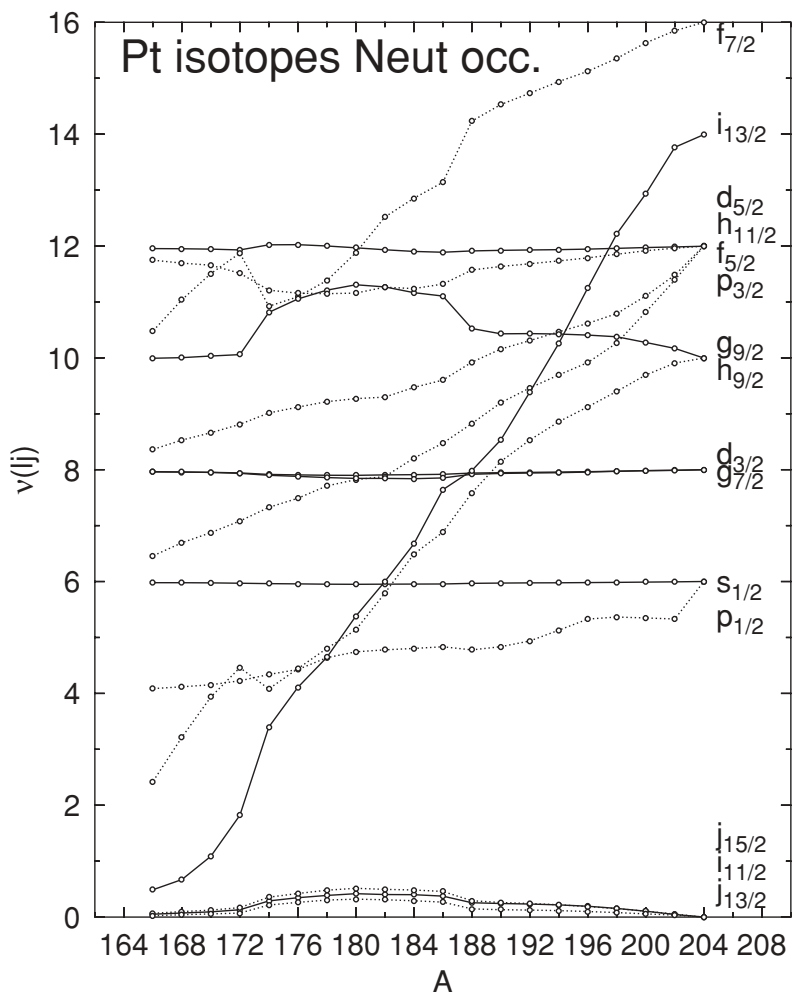

FIG. 10. Spherical occupancies in the proton and neutron ground-state wave functions for the isotopes ${ }^{166-204} \mathrm{Pt}$. For details, see the main text. 
including ${ }^{166-172} \mathrm{Pt}$ and ${ }^{188-204} \mathrm{Pt}$ and the strong deformation regime including the nuclei ${ }^{174-186} \mathrm{Pt}$. It is noteworthy that in each of the regimes, the proton occupancies remain rather constant irrespective of the $\gamma$ deformation and even the specific value of the $Q$ deformation parameter. The strong deformation regime differs from the weak deformation one in that the $d_{5 / 2}$, $d_{3 / 2}$, and $h_{11 / 2}$ orbitals lose occupancy in favor of the $f_{7 / 2}, f_{5 / 2}$, and $h_{9 / 2}$. This rearrangement of the occupancies is mainly due to the smearing out of the Fermi surface as a consequence of the increasing proton pairing correlations (see Fig. 6) and to a lesser extent to the quadrupole interaction among orbits that can transfer particles from one orbit to another. Given the shift of occupancies from the $d_{5 / 2}, d_{3 / 2}$, and $h_{11 / 2}$ to the $f_{7 / 2}, f_{5 / 2}$, and $h_{9 / 2}$ orbitals, we can interpret the well-deformed ground state of ${ }^{174-186} \mathrm{Pt}$ as a multiparticle-multihole excitation out of a reference spherical ground state. The number of particles exchanged in this kind of spherical shell model language is between two and four according to the results shown in the left panel of Fig. 10.

In the case of neutrons, shown on the right panel of Fig. 10, as the neutron number increases, we are occupying orbitals belonging to the $N=5$ negative parity major shell and the positive parity intruder $i_{13 / 2}$. As a consequence of deformation, the spherical orbitals are mixed up, and therefore placing two particles in a given deformed orbital by no means implies placing two particles in a spherical orbit. The two particles will be distributed among all the components of the deformed orbital when expressed in the spherical basis. As a consequence, the behavior of the spherical occupancies with neutron number is more or less linear in the whole interval and for all the orbitals involved. The only noticeable deviation from this trend takes place when entering the strong deformation regime where the $f_{7 / 2}, h_{9 / 2}$, and $h_{11 / 2}$ orbitals lose particles in favor of the high- $j$ orbitals $i_{13 / 2}, i_{11 / 2}, j_{13 / 2}$, and $j_{15 / 2}$. Also the $g_{9 / 2}$ orbital gets more particles (through the coupling to the $N=6$ orbital). This change in occupancies can be mostly attributed to the quenching of neutron pairing correlations in the ground state of the strongly deformed isotopes ${ }^{174-186} \mathrm{Pt}$. When the weak deformation regime is entered at $A=188$, a much smoother behavior of the spherical occupancies is recovered. This, together with the smooth behavior of proton occupancies, is quite unexpected, as in the weak deformation regime the different isotopes have a variety of ground-state deformations ranging from prolate to triaxial to oblate. The conclusion is that the occupancies are more sensitive to the magnitude of the deformation $Q$ than to the $\gamma$ degree of freedom.

The proton levels closest to the Fermi level are the $s_{1 / 2}$, $h_{11 / 2}, d_{3 / 2}$, and $d_{5 / 2}$ and therefore are the ones expected to strongly interact with the neutron spin orbit partner according to the FP mechanism. The more effective orbitals are those with high $j$, and therefore the proton $h_{11 / 2}$ is expected to strongly interact with the neutron $h_{9 / 2}$, which is empty at the beginning of the chain and gets steadily occupied as more neutrons are added. Also a strong interaction is expected with the neutron $i_{13 / 2}$ orbital that shares with the $h_{9 / 2}$ one the occupation pattern as a function of $N$. It is also noteworthy to point out how proton $h_{9 / 2}$, that should be empty according to the spherical shell model, gets some occupancy for the well-deformed nuclei ${ }^{174-186} \mathrm{Pt}$ as a consequence of the smearing out of the Fermi surface, which allows those orbitals to interact via FP with the neutrons $h_{11 / 2}$ and $g_{9 / 2}$. It could be argued that the proton $h_{11 / 2}$ orbital is losing two particles in the strong deformation regime, and this could imply a strong impact in its interaction with its neutron spin-orbit partner. This would be true if the $h_{11 / 2}$ orbital were occupied by a small amount of neutrons (of the order of two), as this would imply completely emptying out the orbital, but bear in mind that there are ten neutrons at the beginning of the region under consideration.

\section{CONCLUSIONS}

In this paper we have studied the evolution of the groundstate nuclear shapes in a series of Pt isotopes ranging from $N=$ $88(A=166)$ up to $N=126(A=204)$, covering practically one complete major shell. The study has been performed within the self-consistent HFB approach based on the D1S [52] and the recent D1N [74] and D1M [75] parametrizations of the Gogny interaction, and we have included, in addition to the axially symmetric limit, the triaxial degrees of freedom $\beta$ and $\gamma$.

From the analysis of the axially symmetric limit, we conclude that a sudden prolate to oblate shape change occurs around $N=110(A=188)$ for all the Gogny parametrizations considered. This result is also in agreement with those obtained either with Skyrme forces or from relativistic mean field calculations $[8,30,61,63]$. On the other hand, when triaxiality is taken into account, the picture that finally emerges is that of smooth transitions between the different shape regimes. We find that the absolute minimum of the PESs for the Pt isotopes evolves from prolate shapes with increasing values of their quadrupole moments $Q$ in the lighter isotopes $A=$ 166-182 (with the exception of $A=172-176$, which exhibit a tendency to triaxiality), to triaxial $\gamma$-soft in the intermediate isotopes with $A=184-196$, and to oblate shapes in the most neutron-rich isotopes $A=198-202$. Finally, the isotope ${ }^{204} \mathrm{Pt}$ becomes spherical. By analyzing PECs and the $Q-\gamma$ landscapes, we observe that the (axial) prolate and oblate minima, well separated by high-energy barriers in the $\beta$ degree of freedom, are softly linked along the $\gamma$ direction. Indeed, most of the secondary axial minima become saddle points when the $\gamma$ degree of freedom is included in the analysis. Pairing energies and Thouless-Valatin moments of inertia have also been analyzed as functions of the number of neutrons, finding some correlation with the evolution of the quadrupole deformation $Q$. Such a correlation, on the other hand, is not so evident in the case of the $\gamma$ angle.

We consider as very positive the similarity between the mean field ground-state properties predicted for the considered $\mathrm{Pt}$ chain with the most recent versions of the Gogny interaction (i.e., D1S, D1N, and D1M) employed in the present study. On one hand, the results give us confidence concerning the robustness of the predictions against the details of the particular effective Gogny interaction used. The robustness is reinforced when the trend of our calculations is compared with the ones obtained with Skyrme forces $[8,63]$ and other theoretical approaches [3,22,33,93], which are again very similar. On the other hand, our results also point to the fact that the new incarnations D1N and D1M of the Gogny interaction, based on fitting protocols more in the 
direction of astrophysical applications [74,75], essentially keep the predictive power of the Gogny-D1S force [52] already considered as a (standard) global force.

We have analyzed the proton and neutron SPEs as functions of both axial and triaxial deformation parameters in some illustrative examples. The analysis has been done in terms of the density of levels around the Fermi surface (Jahn-Teller effect [88]) and the Federman-Pittel mechanism [98]. Our discussion has also been illustrated with the calculation of the spherical occupancies in the ground-state wave functions of the considered Pt nuclei. As a result, we obtain a qualitative understanding of the emergence of deformed configurations with two main ingredients, which are (i) the energy gaps that appear at different deformations in the SPEs of neutrons when the Fermi level $\lambda_{N}$ crosses different regions and (ii) the special role of the overlap between the proton $1 h_{11 / 2}$ and the neutron $1 i_{13 / 2}$ orbitals.

The study of the low-lying excitation spectra and transition rates in conjunction with shape transitions would require extending the present mean field approach to take into account correlations related to the restoration of broken symmetries and fluctuations of collective variables. The restoration of broken symmetries would imply, among others, triaxial projections to restore rotational symmetry, and this is a very difficult and delicate issue with realistic forces [102-105]. These difficulties lead us to consider, instead of the exact projection, some kind of approximation to it that could eventually end up in a kind of collective Bohr Hamiltonian [82,94-96] with deformation-dependent parameters. Upon completion of this work, a preprint has appeared [87] dealing with the calculation of $2^{+}$excitation energies in the framework of the five-dimensional Bohr Hamiltonian method with parameters extracted from a microscopic mean field calculation with the Gogny D1S force. As a consequence of the number of nuclei considered (around two thousand) in that calculation, their analysis of the mean field results is not as exhaustive as ours.

\section{ACKNOWLEDGMENTS}

This work was partly supported by MEC (Spain) under Contract FPA2007-66069, MICINN (Spain) under Contract FIS2008-01301, the Consolider-Ingenio 2010 program CPAN (Spain) under Contract CSD2007-00042, and Junta de Andalucía (Spain) under Contract P07-FQM-02962. The first steps of this work were undertaken in the framework of the FIDIPRO program (Academy of Finland and University of Jyväskylä) and one of us (R.R.) thanks Profs. J. Dobaczewski, J. Äysto, and R. Julin and the experimental teams of the University of Jyväskylä (Finland) for their warm hospitality and encouraging discussions. We thank Prof. S. Goriely (Université Libre de Bruxelles, Belgium) for making the parametrization Gogny-D1M available to us prior to publication and also for valuable suggestions. Valuable suggestions from Prof. K. Heyde are also acknowledged.
[1] A. Bohr and B. R. Mottelson, Nuclear Structure (Benjamin, New York, 1969 and 1975), Vols. I and II.

[2] P. Ring and P. Schuck, The Nuclear Many-Body Problem (Springer, New York, 1980).

[3] J. L. Wood, K. Heyde, W. Nazarewicz, M. Huyse, and P. Van Duppen, Phys. Rep. 215, 101 (1992).

[4] T. R. Werner, J. Dobaczewski, M. W. Guidry, W. Nazarewicz, and J. A. Sheikh, Nucl. Phys. A578, 1 (1994).

[5] M. Bender, P.-H. Heenen, and P.-G. Reinhard, Rev. Mod. Phys. 75, 121 (2003).

[6] S. Cwiok, P.-H. Heenen, and W. Nazarewicz, Nature (London) 433, 705 (2005).

[7] L. M. Robledo, R. R. Rodríguez-Guzmán, and P. Sarriguren, Phys. Rev. C 78, 034314 (2008).

[8] P. Sarriguren, R. Rodríguez-Guzmán, and L. M. Robledo, Phys. Rev. C 77, 064322 (2008).

[9] R. Rodríguez-Guzmán and P. Sarriguren, Phys. Rev. C 76, 064303 (2007).

[10] R. Julin, K. Helariutta, and M. Muikku, J. Phys. G 27, R109 (2001).

[11] G. D. Dracoulis, A. E. Stuchbery, A. P. Byrne, A. R. Poletti, S. J. Polotti, J. Gerl, and R. A. Bark, J. Phys. G 12, L97 (1986); G. D. Dracoulis et al., Phys. Rev. C 44, R1246 (1991).

[12] P. M. Davidson et al., Nucl. Phys. A657, 219 (1999).

[13] C. Y. Wu et al., Nucl. Phys. A607, 178 (1996).

[14] Zs. Podolyák et al., Phys. Lett. B491, 225 (2000).

[15] M. Pfützner et al., Phys. Rev. C 65, 064604 (2002).

[16] M. Caamaño et al., Eur. Phys. J. A 23, 201 (2005).

[17] A. N. Andreyev et al., Nature (London) 405, 430 (2000).

[18] M. Bender, T. Cornelius, G. A. Lalazissis, J. A. Maruhn, W. Nazarewicz, and P.-G. Reinhard, Eur. Phys. J. A 14, 23 (2002).
[19] M. Bender, G. F. Bertsch, and P.-H. Heenen, Phys. Rev. Lett. 94, 102503 (2005).

[20] T. Grahn et al., Nucl. Phys. A801, 83 (2008).

[21] S. L. King et al., Phys. Lett. B443, 82 (1998).

[22] B. Cederwall et al., Phys. Lett. B443, 69 (1998).

[23] D. Seweryniak et al., Phys. Rev. C 58, 2710 (1998).

[24] F. G. Kondev et al., Phys. Rev. C 61, 044323 (2000).

[25] F. Le Blanc et al., Phys. Rev. C 60, 054310 (1999).

[26] D. T. Joss et al., Phys. Rev. C 74, 014302 (2006).

[27] F. Soramel et al., Eur. Phys. J. A 4, 17 (1999).

[28] D. G. Popescu et al., Phys. Rev. C 55, 1175 (1997).

[29] Y. Xu, K. S. Krane, M. A. Gummin, M. Jarrio, J. L. Wood, E. F. Zganjar, and H. K. Carter, Phys. Rev. Lett. 68, 3853 (1992).

[30] J. Sauvage-Letessier, P. Quentin, and H. Flocard, Nucl. Phys. A370, 231 (1981).

[31] A. Ansari, Phys. Rev. C 33, 321 (1986).

[32] P. Bonche, S. J. Krieger, P. Quentin, M. S. Weiss, J. Meyer, M. Meyer, N. Redon, H. Flocard, and P.-H. Heenen, Nucl. Phys. A500, 308 (1989).

[33] R. Bengtsson, T. Bengtsson, J. Dudek, G. Leander, W. Nazarewicz, and J. Z. Zhang, Phys. Lett. B183, 1 (1987).

[34] P. Möller, J. R. Nix, W. D. Myers, and W. J. Swiatecki, At. Data Nucl. Data Tables 59, 185 (1995).

[35] P. Möller, R. Bengtsson, B. G. Carlsson, P. Olivius, T. Ichikawa, H. Sagawa, and A. Iwamoto, At. Data Nucl. Data Tables 94, 758 (2008).

[36] Table of Isotopes: 1999 Update with CD-ROM, 8th ed., edited by R. B. Firestone, C. M. Baglin, and S. Y. Frank Chu (Wiley, New York, 1999).

[37] Th. Hilberath et al., Z. Phys. A 342, 1 (1992).

[38] J. Dechargé and D. Gogny, Phys. Rev. C 21, 1568 (1980). 
[39] F. R. May, V. V. Pashkevich, and S. Frauendorf, Phys. Lett. B68, 113 (1997).

[40] R. Bengtsson and W. Nazarewicz, Z. Phys. A 334, 269 (1989).

[41] W. Nazarewicz, Phys. Lett. B305, 195 (1993).

[42] S. Yoshida, S. K. Patra, N. Takigawa, and C. R. Praharaj, Phys. Rev. C 50, 1398 (1994).

[43] S. K. Patra, S. Yoshida, N. Takigawa, and C. R. Praharaj, Phys. Rev. C 50, 1924 (1994).

[44] T. Niksic, D. Vretenar, P. Ring, and G. A. Lalazissis, Phys. Rev. C 65, 054320 (2002).

[45] G. A. Lalazissis, S. Karatzikos, R. Fossion, D. Pena Arteaga, A. V. Afanasjev, and P. Ring, Phys. Lett. B671, 36 (2009).

[46] P.-H. Heenen, A. Valor, M. Bender, P. Bonche, and H. Flocard, Eur. Phys. J. A 11, 393 (2001).

[47] J. Meyer, P. Bonche, M. S. Weiss, J. Dobaczewski, H. Flocard, and P.-H. Heenen, Nucl. Phys. A588, 597 (1995).

[48] N. A. Smirnova, P.-H. Heenen, and G. Neyens, Phys. Lett. B569, 151 (2003).

[49] P. Sarriguren, O. Moreno, R. Alvarez-Rodríguez, and E. M. de Guerra, Phys. Rev. C 72, 054317 (2005).

[50] O. Moreno, P. Sarriguren, R. Alvarez-Rodríguez, and E. Moya de Guerra, Phys. Rev. C 73, 054302 (2006).

[51] J. L. Egido, L. M. Robledo, and R. R. Rodríguez-Guzmán, Phys. Rev. Lett. 93, 082502 (2004).

[52] J. F. Berger, M. Girod, and D. Gogny, Nucl. Phys. A428, 23c (1984).

[53] T. Duguet, M. Bender, P. Bonche, and P.-H. Heenen, Phys. Lett. B559, 201 (2003).

[54] M. Bender, P. Bonche, T. Duguet, and P.-H. Heenen, Phys. Rev. C 69, 064303 (2004).

[55] R. R. Rodríguez-Guzmán, J. L. Egido, and L. M. Robledo, Phys. Rev. C 69, 054319 (2004).

[56] J. P. Delaroche et al., Phys. Rev. C 50, 2332 (1994).

[57] W. Nazarewicz, M. A. Riley, and J. D. Garrett, Nucl. Phys. A512, 61 (1990).

[58] C. Wheldon et al., Phys. Rev. C 63, 011304(R) (2000).

[59] Z. Naik, B. K. Sharma, T. J. Jha, P. Arumugam, and S. K. Patra, Pramana 62, 827 (2004).

[60] R. Fossion, D. Bonatsos, and G. A. Lalazissis, Phys. Rev. C 73, 044310 (2006).

[61] M. M. Sharma and P. Ring, Phys. Rev. C 46, 1715 (1992).

[62] P. D. Stevenson, M. P. Brine, Zs. Podolyak, P. H. Regan, P. M. Walker, and J. R. Stone, Phys. Rev. C 72, 047303 (2005).

[63] L. M. Robledo, R. Rodríguez-Guzmán, and P. Sarriguren, J. Phys. G 36, 115104 (2009).

[64] P. D. Duval and B. R. Barret, Nucl. Phys. A376, 213 (1982).

[65] J. Pakarinen et al., Phys. Rev. C 75, 014302 (2007).

[66] K. Heyde, J. Jolie, J. Moreau, J. Ryckebusch, M. Waroquier, P. Van Duppen, M. Huyse, and J. L. Wood, Nucl. Phys. A466, 189 (1987).

[67] K. Heyde, J. Schietse, and C. De Coster, Phys. Rev. C 44, 2216 (1991).

[68] A. M. Oros, K. Heyde, C. De Coster, B. Decroix, R. Wyss, B. R. Barrett, and P. Navratil, Nucl. Phys. A645, 107 (1999).

[69] V. Hellemans, R. Fossion, S. D. Baerdemacker, and K. Heyde, Phys. Rev. C 71, 034308 (2005).

[70] V. Hellemans, S. De Baerdemacker, and K. Heyde, Phys. Rev. C 77, 064324 (2008).

[71] J. E. Garcia-Ramos and K. Heyde, Nucl. Phys. A825, 39 (2009).

[72] I. O. Morales, A. Frank, C. E. Vargas, and P. Van Isacker, Phys. Rev. C 78, 024303 (2008).
[73] D. Vretenar, A. V. Afanasjev, G. A. Lalazissis, and P. Ring, Phys. Rep. 409, 101 (2005).

[74] F. Chappert, M. Girod, and S. Hilaire, Phys. Lett. B668, 420 (2008).

[75] S. Goriely, S. Hilaire, M. Girod, and S. Péru, Phys. Rev. Lett. 102, 242501 (2009).

[76] J. F. Berger, M. Girod, and D. Gogny, Nucl. Phys. A502, 85c (1989).

[77] C. R. Chinn, J. F. Berger, D. Gogny, and M. S. Weiss, Phys. Rev. C 45, 1700 (1992).

[78] J. L. Egido and L. M. Robledo, Phys. Rev. Lett. 85, 1198 (2000).

[79] J. L. Egido and L. M. Robledo, Nucl. Phys. A494, 85 (1989).

[80] M. Girod, J. P. Delaroche, D. Gogny, and J. F. Berger, Phys. Rev. Lett. 62, 2452 (1989).

[81] J. L. Egido and L. M. Robledo, Phys. Rev. Lett. 70, 2876 (1993).

[82] G. F. Bertsch, M. Girod, S. Hilaire, J.-P. Delaroche, H. Goutte, and S. Péru, Phys. Rev. Lett. 99, 032502 (2007).

[83] S. Péru, J. F. Berger, and P. F. Bortignon, Eur. Phys. J. A 26, 25 (2005).

[84] R. R. Rodríguez-Guzmán, J. L. Egido, and L. M. Robledo, Nucl. Phys. A709, 201 (2002).

[85] R. R. Rodríguez-Guzmán, J. L. Egido, and L. M. Robledo, Phys. Rev. C 65, 024304 (2002).

[86] S. Hilaire and M. Girod, Eur. Phys. J. A 33, 237 (2007).

[87] J.-P. Delaroche, M. Girod, J. Libert, H. Goutte, S. Hilaire, S. Péru, N. Pillet, and G. F. Bertsch, Phys. Rev. C 81, 014303 (2010).

[88] P.-G. Reinhard and E. W. Otten, Nucl. Phys. A420, 173 (1984).

[89] J. L. Egido, J. Lessing, V. Martin, and L. M. Robledo, Nucl. Phys. A594, 70 (1995).

[90] N. Tajima, S. Takahara, and N. Onishi, Nucl. Phys. A603, 23 (1996)

[91] E. Chabanat, P. Bonche, P. Haensel, J. Meyer, and R. Schaeffer, Nucl. Phys. A635, 231 (1998).

[92] J. Terasaki, P.-H. Heenen, H. Flocard, and P. Bonche, Nucl. Phys. A600, 371 (1996).

[93] B. Cederwall et al., Z. Phys. A 337, 283 (1990).

[94] J. Libert, M. Girod, and J.-P. Delaroche, Phys. Rev. C 60, 054301 (1999).

[95] T. Niksic, Z. P. Li, D. Vretenar, L. Próchniak, J. Meng, and P. Ring, Phys. Rev. C 79, 034303 (2009).

[96] L. Próchniak and S. G. Rohoziński, J. Phys. G 36, 123101 (2009).

[97] D. L. Hill and J. A. Wheeler, Phys. Rev. 89, 1102 (1953).

[98] P. Federman and S. Pittel, Phys. Lett. B69, 385 (1977); Phys. Rev. C 20, 820 (1979).

[99] W. Nazarewicz, Nucl. Phys. A574, 27c (1994).

[100] I. Hamamoto and B. R. Mottelson, Phys. Rev. C 79, 034317 (2009).

[101] R. Fossion, Rev. Mex. Fis. S54, 42 (2008).

[102] M. Bender and P.-H. Heenen, Phys. Rev. C 78, 024309 (2008).

[103] D. Lacroix, T. Duguet, and M. Bender, Phys. Rev. C 79, 044318 (2009).

[104] M. Bender, T. Duguet, and D. Lacroix, Phys. Rev. C 79, 044319 (2009).

[105] T. Duguet, M. Bender, K. Bennaceur, D. Lacroix, and T. Lesinski, Phys. Rev. C 79, 044320 (2009). 